\title{
Introduction of a Complex Reaction Time Tester Instrument
}

\author{
Roland Reginald Zana1*, Ambrus Zelei² \\ 1 Department of Applied Mechanics, Faculty of Mechanical Engineering, Budapest University of Technology and Economics \\ 1111 Budapest, Muegyetem rkp. 5., Hungary \\ 2 MTA-BME Lendület Human Balancing Research Group, 1111 Budapest, Muegyetem rkp. 5., Hungary \\ *Corresponding author, e-mail: zana_r@mm.bme.hu
}

Received: 28 January 2019, Accepted: 06 November 2019, Published online: 08 December 2019

\begin{abstract}
The reaction time, which is also referred as reflex delay in the literature, is an important factor in human balancing, since reaction time highly affects the ability of self stabilization. Increased reaction time delay may cause dangerous fall-over accidents related to elderly people. Reaction time depends on age, health, everyday activities, the general and actual physical and mental state of the individual and the environmental conditions.

The reaction time is considered as a parameter in many of the mathematical models of the neural processes in human balancing. It is beneficial in many cases to estimate the reaction time based on experimental data.

The present paper introduces the prototype of a complex reaction time tester instrument. The novelty of the instrument is that the reaction time can be measured in various combinations of sensory organs and reaction movements. The reaction time is defined as the time duration in between the initial time instant of the stimulus of the sensory organs (input signal) and the onset of the response that is typically indicated by a button or a pedal. Another novelty is that the instrument is free of any uncertain time delay, which is not the case for several instruments available.

Usually, human simple reaction time is considered to be roughly about $200 \mathrm{~ms}$. The shortest (aural) reaction time for skilled athletes is $85 \mathrm{~ms}$. In our measurements the shortest reaction time was $97 \mathrm{~ms}$, and the mean about $190 \mathrm{~ms}$ in simple reaction cases. So our collected experimental data are in agreement with the literature.
\end{abstract}

Keywords

human reaction time delay, neural models, human balancing, experimental devices

\section{Introduction}

The reaction time is an important factor which affects humans' motor control. In general, the sensory organs send signals to the brain. These signals are processed and the brain makes decisions on the modification of the body posture and the muscle contraction. Then, the musculoskeletal system receives the specified signals from the brain, which causes the correction of the motion [1-3]. As an analogy, we mention the feedback control systems from the engineering field: the sensory signals - such as acceleration or servo drive encoder data - are usually processed by computers and the control signals are sent to the actuators. The sensing-actuation loop takes several milliseconds, which is referred as reaction time in case of humans and animals [1, 2] and referred as control delay or feedback delay in case of robots and other computer controlled dynamic systems $[4,5]$.
When the neural processes behind the human balancing are studied or modeled, the humans' reaction time is especially considerable. For instance, the stability conditions of standing still are highly affected not only by the sensory dead zones but also by the reaction time delay. Measurements show [2] that a certain chaotic-like oscillation of the body can be observed even during standing still. This oscillation is in relation with the reaction time. The underlying dynamic model of postural balancing is usually the inverted pendulum or the inverted double pendulum model [6], of which the otherwise unstable upward position is stabilized by a controller. In case of postural balancing, the reaction time is determined by the processing and travelling time of signals from the vestibular system to the brain; the processing and travelling time of visual information from the retina to the brain; the processing and travelling time of the signals 
related to the kinesthetic sense; and the travelling time of signals through the nervous system to the motor neurons that activate basically the leg and torso muscles.

In general, we can say that reaction time depends on many factors such as age, health, everyday activities, the general and actual physical and mental condition of the individual and the environmental conditions [7-9]. The reaction time depends on the specific sensory organs; the length of the nervous tissue in which the signals are transferred; and muscle contraction time. Consequently, the accurate modeling of human neural processes and human motor system demands the measurement of the reaction time delay of the combination of specific sensory organs and specific parts of the musculoskeletal system. The numerous combinations of the resulting reaction time values may be present in the human balancing models.

The measurement of reaction time delay has several other important aspects. Humans' reaction time has an influence on the maximum allowable lag by designing human machine interfaces to remain controllable. Lag here is the delay between input action and output response of any machine. The related results in paper [10] are important regarding the design of human-machine interfaces, e.g. computer mouse and virtual reality devices. Reaction time measurements are also important when the effects of drugs are studied; the positive effects of caffeine are studied in [11]. According to [12], the measurement of reaction time can be also used as an early sign of cognitive decline.

Several reaction time testers from the literature are listed in Section 1.1; however most of them are developed for the measurement of a specific combination of a sensory organ and a reaction movement, their main properties are collected in Table 1. In Section 2, we detail the design and the capabilities of our complex reaction time tester (CRTT), which is used for measuring the reaction time in several combinations of sensory organs and reaction movements. Sections 3-5 present new experimental data, which illustrates the capabilities of the CRTT; additionally, the measured values are validated by comparing the results with the literature.

\subsection{Reaction time testers from the literature}

In most of the cases, the reaction time measurement devices are related with specific sport sciences. The currently existing devices can be categorized into different groups. The first point of view I) is the scale of the required reaction movement. There exist such test devices, which analyze the reaction and the coordination abilities and the agility of the whole human body, such as the instruments shown in Fig. 1 [13], Fig. 2 [14] and Fig. 3 [15]. In the case of these devices, the reaction time is not clearly assigned for specific sensory organ - reaction motion pairs; they rather collect information about the mixture of the different reaction times.

In most of the cases, the task is to simply press or release a button or a pedal in a few $\mathrm{cm}$ range of the finger or feet [16-18]. The instrument form [16] called American Educational (Hubbard Scientific 6027) Reaction Timer is shown in Fig. 4 [16] left. The instrument measures the time range between the beep and the pushing of the button in hundredths of a second. On the right panel of Fig. 4, the

Table 1 Comparison of reaction time testers

\begin{tabular}{|c|c|c|c|c|c|}
\hline & $\begin{array}{l}\text { Scale of the required reaction } \\
\text { movement - Reaction/Input }\end{array}$ & $\begin{array}{l}\text { Source of the } \\
\text { stimulus }\end{array}$ & $\begin{array}{l}\text { Complex reaction } \\
\text { measurement possibility }\end{array}$ & $\begin{array}{l}\text { Mobility and } \\
\text { versatility }\end{array}$ & $\begin{array}{l}\text { Additional time } \\
\text { delay possibility }\end{array}$ \\
\hline Batak & $\begin{array}{l}\text { fingers - whole body } \\
\text { movement }\end{array}$ & light & limited & yes & no data \\
\hline Fitlight Trainer & $\begin{array}{l}\text { fingers - whole body } \\
\text { movement }\end{array}$ & light & limited & yes & no data \\
\hline AFL & fingers & light & no & yes & \\
\hline Gadgets & fingers - touch & light/sound & no & yes & miliseconds \\
\hline Moart & fingers- touch & light/sound & yes & yes & \\
\hline Ruler like & fingers/palm - grip & visual & no & yes & miliseconds \\
\hline DPA-1 & $\begin{array}{l}\text { upper/lower limb - } \\
\text { coordination }\end{array}$ & visual & yes & no & no data \\
\hline Photo diode detector & leg lifting & sound & no & yes & no data \\
\hline Mats & leg lifting & sound & no & moderate & no data \\
\hline Computer/Tablet/Smart phone & fingers-touch & light/sound & yes & yes & $\begin{array}{l}\text { high - tens of } \\
\text { miliseconds }\end{array}$ \\
\hline CRTT & fingers/leg - touch & light/sound & yes & yes & nanoseonds \\
\hline
\end{tabular}



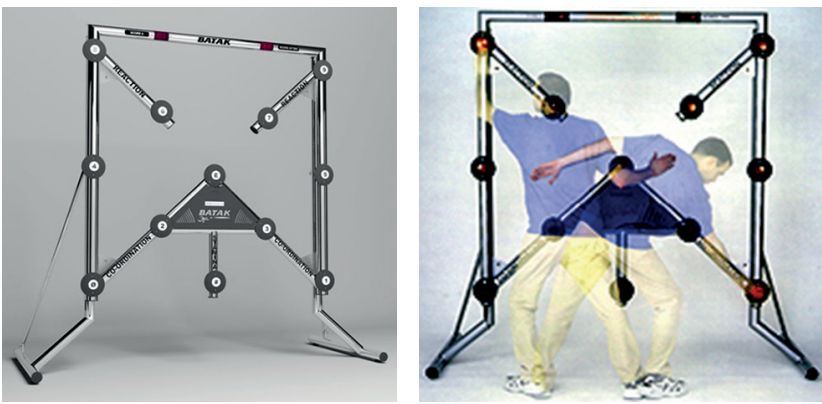

Fig. 1 The Batak is a system for improve fitness and reaction time [13]

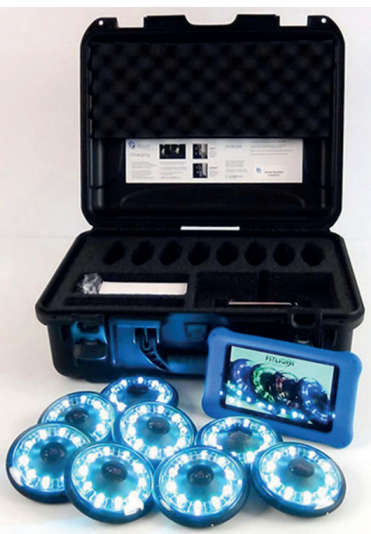

Fig. 2 The Fitlight Trainer wireless touch pads [14]

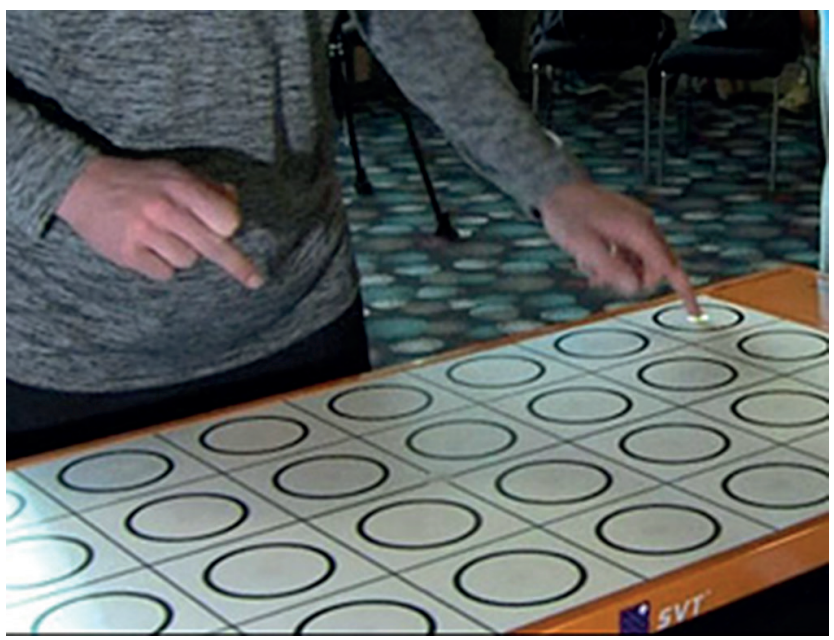

Fig. 3 The AFL Reaction Time test for of eye-hand coordination and reaction time measurements [15]

Milli-Speed Reaction Timer is shown. Here the stimulus is given by a flashing light and the reaction is the pushing of a rubber button. The Lafayette Institute's MOART device $[17,18]$ (shown in Fig. 5) is a widely applicable device. This device can be used for simple and complex reaction time measurements with buttons and pedals as input, and lights and tones as stimulus combined arbitrarily. In many cases, such as in the case of the instrument [19] shown in Fig. 6, the task of the subject is to catch a ruler like device
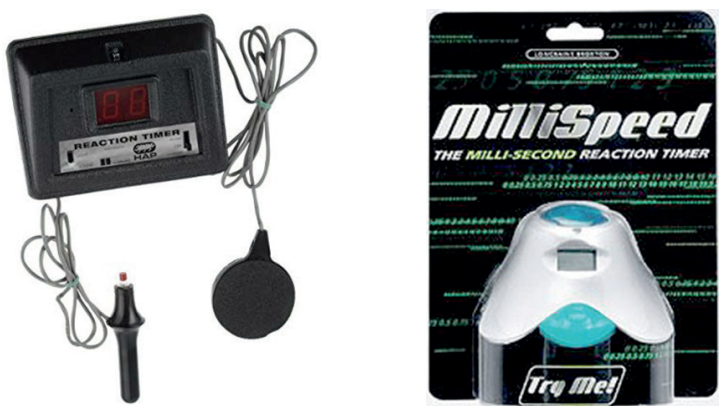

Fig. 4 Reaction Time Gadgets [16]

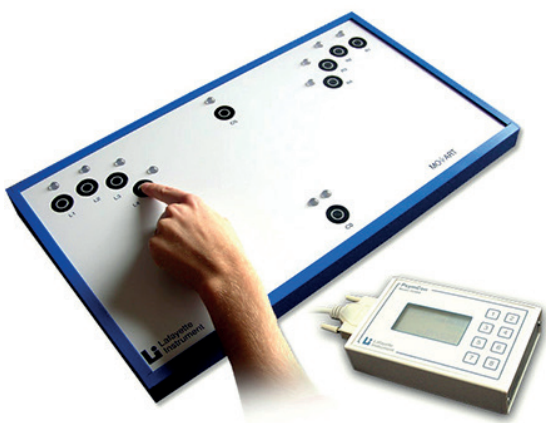

Fig. 5 Lafayette Institute's MOART device [18]
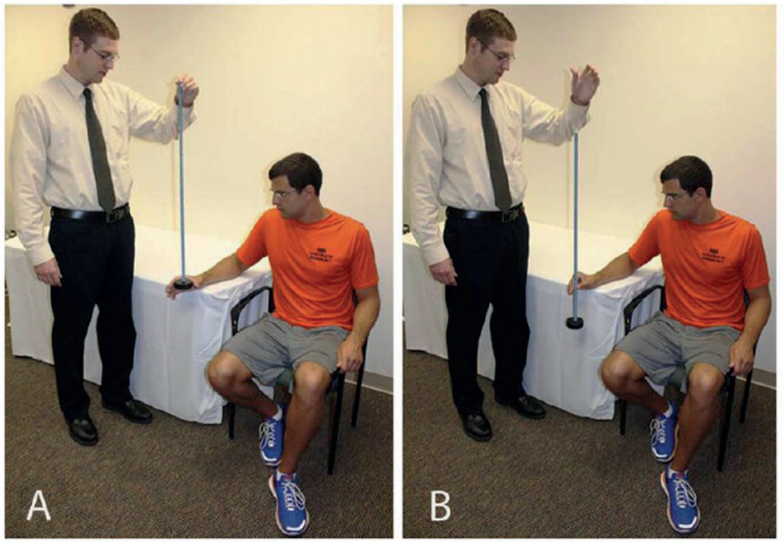

Fig. 6 A ruler like reaction time measurement device [19]

after its random time release. This also requires small scale motion of the hand.

The second point of view II) is the source of the stimulus, which can be a flashing light, a beep or even vibration, such as in case of the above explained instruments.

As a third point of view III), the devices are categorized into simple and complex reaction time measurements according to the required reaction; the latter expects decision-making before reacting, such as in [17, 18].

Our forth point of view IV) is the mobility and versatility: the common disadvantage of some devices, such as [20-22], 
is that they operate only in a laboratory as an experimental set-up and most of them can only be applied to a small fraction of stimulus-reaction pairs. Fig. 7 shows a picture of the DPA-1 instrument [20], which was developed for the dynamic analysis of the upper and lower limb movements, while a given point on the display is reached by using the controller handles of the instrument. In [21], a laboratory experiment is presented for the measurement of reaction time of the leg lifting in reaction to a sound stimulus (see Fig. 8 left side). Force sensors are integrated into two mats in the experiment presented in [22] (see Fig. 8 right side).

Our fifth point of view V) is the electronic instrument on which the reaction time tests are implemented. Depending on the instrument, certain or uncertain additional time delay might be involved into the measurements. The tests can run on a computer, a tablet PC or a smart phone [23-25], however the set-ups which use monitors or any kind of digital displays suffer from the unknown latency: the object appear on the screen with unknown

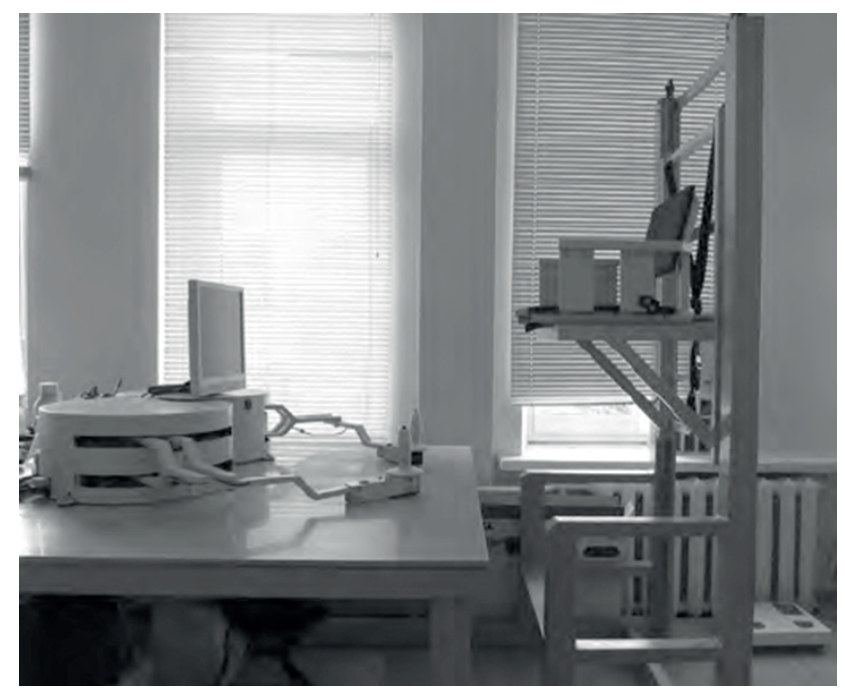

Fig. 7 Analyzer DPA-1 of dynamic upper and lower limb movements [20]

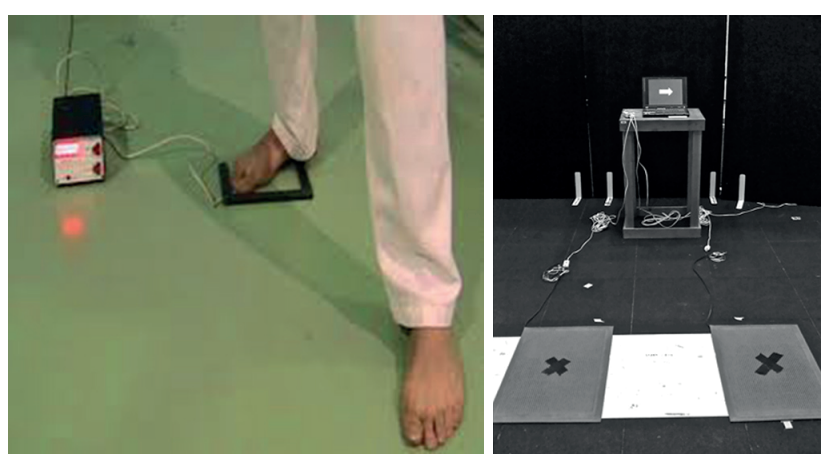

Fig. 8 Left: Leg lifting photo diode detector to sound stimulus [21], right: Mats with force sensor installed for simple and complex reaction time tests [22] delay. The results in [26] shows that the time delay is typically in the range of 30-100 ms depending of the manufacturer and type of the display, and it is also shown that the delay changes stochastically. Regarding to the delay problem, the online tests are the worst, where the server-client communication is an additional stochastic latency. That's why the best solution is a microcontroller unit based device equipped with simple feedback units, such as a flashing led or a beeping speaker and with simple sensors such as a button, touch or a force sensor. Such instrument can provide much more accurate measurements. Our instrument is developed in respect to the issue of additional stochastic delay as it is explained in the specification in Section 2.

\subsection{Goal of the present research}

In this paper we demonstrate a new reaction time tester instrument, which incorporates the benefits of the previous listed devices. The capabilities of the instrument are presented by human benchmark tests.

\section{The complex reaction time tester}

Our newly developed CRTT device is a multifunctional central unit, which is extendable with various peripheral hardware devices for generating stimulus and various components for detecting reaction. The block diagram of the CRTT is shown in Fig. 9. The central unit is a microcontroller based device shown in Fig. 10. Our instrument is significantly cheaper than many of the instruments from the market, such as the instrument in [17]. The central unit communicates with a computer and the periphery contoller via UART on simple USB cable. The central unit requires 9V DC. These are PIC microcontroller unit based devices with external oscillators for high precision and reliability. The user can select the desired test mode and can set the parameters in a MatLab GUI. The measured data transfers directly to the computer and it is processed automatically. Additionally, there is a periphery controller that communicates with the central unit via UART BUS. The task of the periphery unit is the direct control of the stimulus generators (speaker and LED), the collection of reaction data (e.g. from the touch periphery and button periphery) and reaction time calculation. The time resolution of the system is 0.1 milliseconds. The delays of the stimulus generation and the reaction measurement are in the magnitude of tens of nanoseconds; therefore, there is no any unexpected measurement inaccuracy.

The most widely used periphery is the button-light unit (Fig. 11 left side). This unit is capable to generate stimuli by means of three white LEDs, and an RGB LED. The 


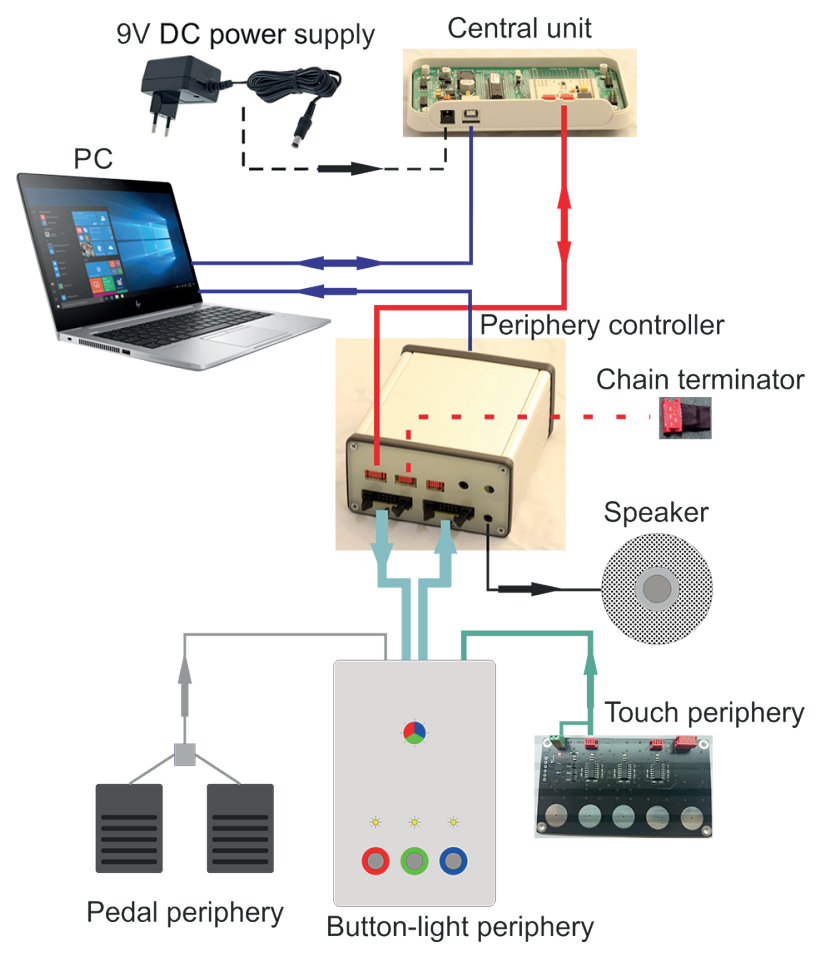

Fig. 9 Block diagram of the CRTT

corresponding buttons can detect the reactions. The input buttons of the button-light peripheral unit are Z15G1307 type snap action micro switches [27] with 250-350 g operation force and maximum $0.4 \mathrm{~mm}$ pre-travel. These buttons can be replaced by touch sensors with almost no operation force and no click/sound of operation. Further peripheral units are the pedal unit for reaction detection and the sound stimulus unit shown in Fig. 12.

Using the current set-up, the following measurements can be carried out. The test types are sorted based on the complexity of the task:

Simple reaction time tests:

- LED - Button: stimulus: green light, reaction: pushing the green button;

- LED - Pedal: stimulus: green light, reaction: pushing the right pedal;

- Periodic LED - Button:

- stimulus: periodic green light with a single deviating time period value, reaction: pushing the green button, trying to accommodate;

- Beep - Button:

stimulus: beep, reaction: pushing the green button;

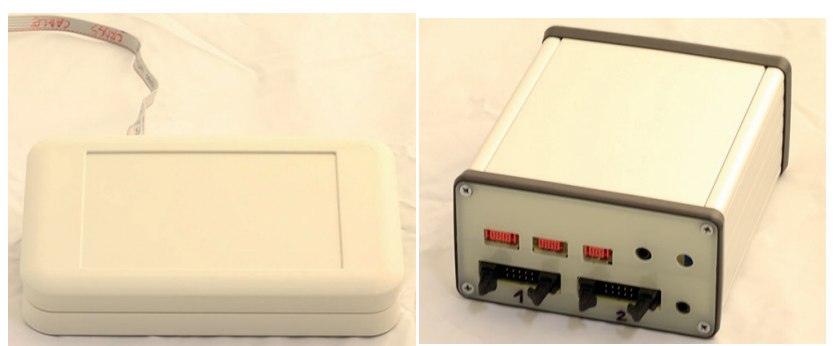

Fig. 10 Left the CRTT central unit, right: the periphery controller
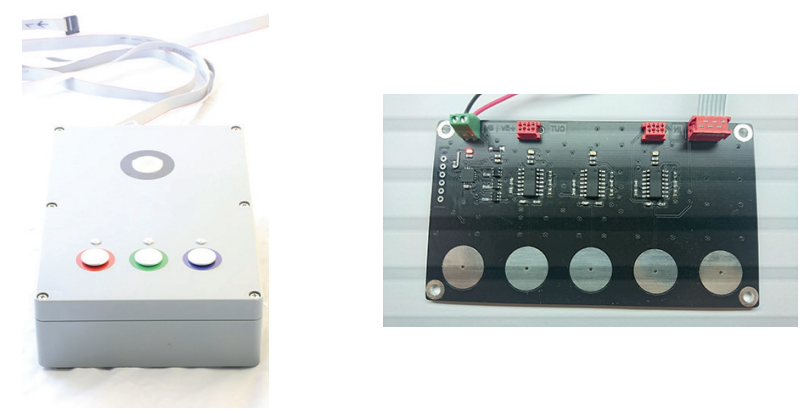

Fig. 11 Left: CRTT button-light peripheral device, right: touch periphery
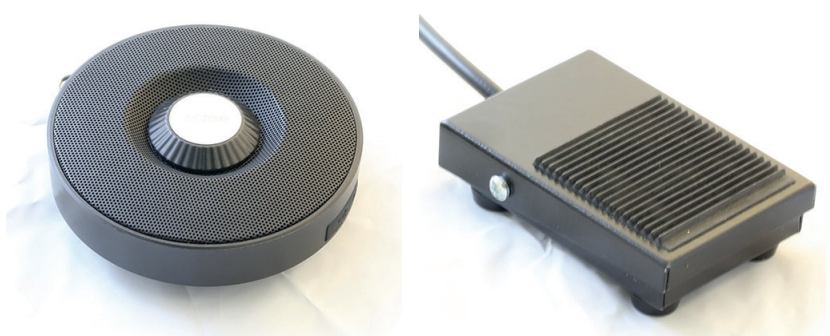

Fig. 12 Left: Peripheral unit for sound stimulus, right: Pedal peripheral unit for reaction detection

- Beep - Pedal:

stimulus: beep,

reaction: pushing the pedal;

- Periodic Beep - Button:

stimulus: periodic beep with a single deviating time period value,

reaction: pushing the green button, trying to accommodate.

Complex reaction time tests:

- RGB:

stimulus: tri-colour light, reaction: pushing the corresponding button;

- Individual: stimulus: light above the buttons, reaction: pushing the corresponding button;

- Pedals:

stimulus: right or left light, reaction: pushing the corresponding pedal with the right or the left foot. 
As Section 3 details, we chose seven test type out of the above listed tests. A database was created by means of laboratory experiments and was analyzed statistically.

\section{Experiments}

10 volunteers were involved in the laboratory experiments, 3 females and 7 males. The research was carried out following the principles of the Declaration of Helsinki. All participants had no self-reported muscular or neurological diseases which could have affected their ability to perform the reaction time measurement tasks. The average age was 27.7 years with the standard deviation: 2.16 years. Before the experiment, the procedure was explained to the subjects and all participants were informed that their participation was voluntary and that they can quit the experiment at any time without stating a reason. After answering all questions, the participants provided written consent to participate in the study prior to participation.

\subsection{Variety of reaction time tests}

The participants were asked to perform the following seven different types of reaction time tests out of the possible tests listed in Section 2:

- Test type 1: LED - Button (single),

- Test type 2: LED - Pedal (single),

- Test type 3: Beep - Button (single),

- Test type 4: Beep - Pedal (single),

- Test type 5: RGB,

- Test type 6: Individual,

- Test type 7: Pedals.

The test types were performed in different order to preclude any carry over effects between the scenarios. The participants performed a practicing session right before each test type. In the learning phase, the participants went through once, exactly the same process as the measurement - except for the random time delay instances. The learning of the task was not a goal; the aim was to become familiar with the CRTT instrument.

The number of trials within a certain test type is programmable. In our study, 10 trials were performed during each test. The reaction time was stored for each trial in case of each test type (for the detailed results, see Section 4). 10 trials were performed also during each learning sessions.

After the appearance of the stimulus, a time range of maximum 1 second is given to the participant to respond. In case of a wrong decision (if complex reaction time test is performed with decision making), it is possible to correct the reaction within one second. Otherwise, in case of a good decision, the stimulus cancels immediately after the reaction.

The range of the random delay - the time period between successive presented stimuli - can be parameterized. Creating random delay is important, because the human can adapt to a regular pace after a few samples, which could influence the measurements (see Sections 3.2 and 4.2). In our measurements, the random delay was set to 0.5-2 second. Results less than 90 ms were considered as impossible values and were excluded based on [7], where $85 \mathrm{~ms}$ is the shortest reported reaction time for skilled athletes. These very short reaction time values suggest that the subject was attempting to anticipate the stimulus; we do not consider this as a reaction.

The test individuals were in sitting position during the measurements. The CRTT was placed on a desk surface. The buttons were actuated with the index finger of the dominant hand, respectively the pedals were pushed with the dominant leg. In case of simple reaction time tests the finger/ foot was continuously in contact with the input button/pedal, while in case of complex tests the individual had to put back the finger to a base position: under the green button.

\subsection{Reaction time in case of rhythmic signals}

As we mentioned earlier, human can adapt to a regular pace of stimuli after a few samples. To investigate this adaptation phenomenon, we performed Periodic Beep Button tests, out of which a single dataset is presented for the demonstration of the capabilities of the CRTT.

In the Periodic Beep - Button test, the participant received beep sounds periodically, and asked to try to push the button in the same time instant of the beep sound. 20 beep sound stimuli were included in a single test. The time period was $1000 \mathrm{~ms}$. A randomly selected stimulus out of the 20 was generated with intentionally longer time period of a random length. The delay (measured from the stimulus) of each reaction was registered.

The accomplishment of the Periodic Beep - Button test required the extension of the control software in MatLab, but no modification of the hardware was required.

\section{Results}

\subsection{Variety of reaction time tests}

In this research 10 subjects were involved. Each participant accomplished 7 test types and 10 trials were generated per each test. Finally we collected a set of $10 \times 7 \times 10=700$ reaction 
time data. Fig. 13 shows the basic statistics of the tests per individuals: the maximum and minimum value, the mean value and the standard deviation. Table 2 sums up the absolute minimum, maximum, mean values and standard deviations for each type of test, summarized for every subject.

The minimum value, the maximum value, the mean value and standard deviation for all 700 measured reaction time data are: $\min (\tau)=0.0966 \mathrm{~s}, \max (\tau)=0.9343 \mathrm{~s}$, mean $(\tau)=0.28368 \mathrm{~s}$ and $\operatorname{SD}(\tau)=0.11902 \mathrm{~s}$ respectively.

We assume that the reaction time changes linearly with the difficulty of the test. We also assume that a certain subject has shorter reaction time in a certain test type than a different subject, if the reaction time is shorter in a different test. Therefore, the possible relationship between
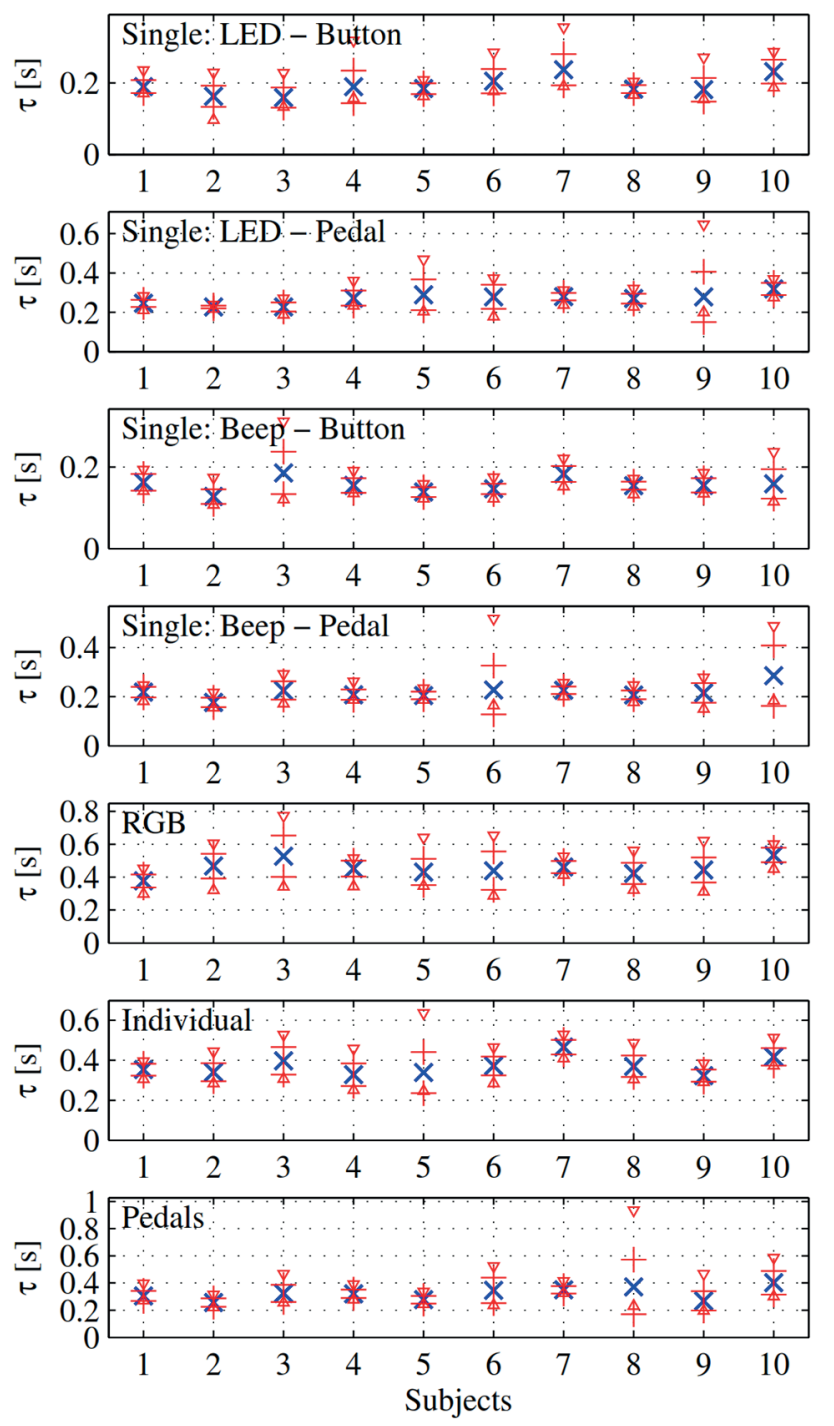

Fig. 13 The maximal value (upper red + ), the minimal value (lower red + ), the mean value (blue $\mathrm{x}$ ) and the standard deviation (triangular shaped red markers) are depicted in case of each subjects (from 1 to 10 on the horizontal axis).
Table 2 The absolute min, max and mean values of the different tests

\begin{tabular}{lcccc}
\hline$\tau[\mathrm{s}]$ & $\min$ & $\max$ & $\operatorname{mean}$ & $\mathrm{SD}$ \\
\hline Single: LED - Button & 0.097 & 0.357 & 0.192 & 0.040 \\
Single: LED - Pedal & 0.179 & 0.647 & 0.268 & 0.062 \\
Single: Beep - Button & 0.108 & 0.311 & 0.157 & 0.030 \\
Single: Beep - Pedal & 0.151 & 0.518 & 0.220 & 0.061 \\
RGB & 0.288 & 0.772 & 0.456 & 0.088 \\
Individual & 0.246 & 0.635 & 0.370 & 0.070 \\
Pedals & 0.197 & 0.934 & 0.322 & 0.095 \\
\hline
\end{tabular}

the different types of tests and the relationship between the different participants was studied with linear correlation calculation. The linear correlations are collected in Table 3 and Table 4. The correlations are visualized in Fig. 14 and Fig. 15. In the diagonal, the autocorrelation results can be found.

In Table 3 and Fig. 14, the numerical values and the subplots respectively show the correlation of the pairs of test types. High correlation between any test types with the indices $i$ and $j$ means that if a participant was fast in a test type $i$, than he/she is fast in test type $j$ too, for any $i-j$ pairs. Each row and column belongs to a certain test type in the same order as in Fig. 13. There were 10 participants; therefore there are 10 points in all subplots. For instance, the

Table 3 Linear correlation between the mean reaction time of tests

\begin{tabular}{cccccccc}
\hline & 1. & 2. & 3. & 4. & 5. & 6. & 7. \\
\hline 1. & 1.000 & 0.754 & 0.290 & 0.682 & 0.116 & 0.677 & 0.655 \\
2. & - & 1.000 & -0.049 & 0.658 & 0.131 & 0.234 & 0.526 \\
3. & - & - & 1.000 & 0.441 & 0.299 & 0.673 & 0.442 \\
4. & - & - & - & 1.000 & 0.511 & 0.562 & 0.755 \\
5. & - & - & - & - & 1.000 & 0.452 & 0.347 \\
6. & - & - & - & - & - & 1.000 & 0.669 \\
7. & - & - & - & - & - & - & 1.000 \\
\hline
\end{tabular}

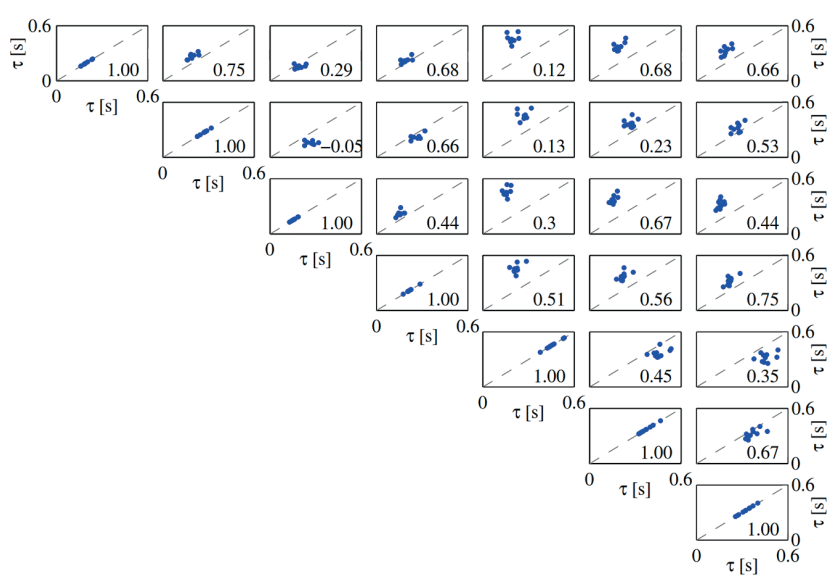

Fig. 14 Results and graphical demonstration of the linear correlation between the tests 
Table 4 Linear correlation between the mean reaction time of participants

\begin{tabular}{ccccccccccc}
\hline & 1. & 2. & 3. & 4. & 5. & 6. & 7. & 8. & 9. & 10. \\
\hline 1. & 1.000 & 0.951 & 0.952 & 0.958 & 0.952 & 0.989 & 0.982 & 0.982 & 0.933 & 0.975 \\
2. & - & 1.000 & 0.978 & 0.976 & 0.976 & 0.956 & 0.938 & 0.918 & 0.985 & 0.958 \\
3. & - & - & 1.000 & 0.956 & 0.932 & 0.939 & 0.928 & 0.923 & 0.950 & 0.944 \\
4. & - & - & - & 1.000 & 0.980 & 0.982 & 0.917 & 0.963 & 0.981 & 0.986 \\
5. & - & - & - & - & 1.000 & 0.970 & 0.927 & 0.933 & 0.992 & 0.969 \\
6. & - & - & - & - & - & 1.000 & 0.958 & 0.986 & 0.952 & 0.993 \\
7. & - & - & - & - & - & - & 1.000 & 0.946 & 0.899 & 0.929 \\
8. & - & - & - & - & - & - & - & 1.000 & 0.910 & 0.971 \\
9. & - & - & - & - & - & - & - & - & 1.000 & 0.961 \\
10. & - & - & - & - & - & - & - & - & - & 1.000 \\
\hline
\end{tabular}

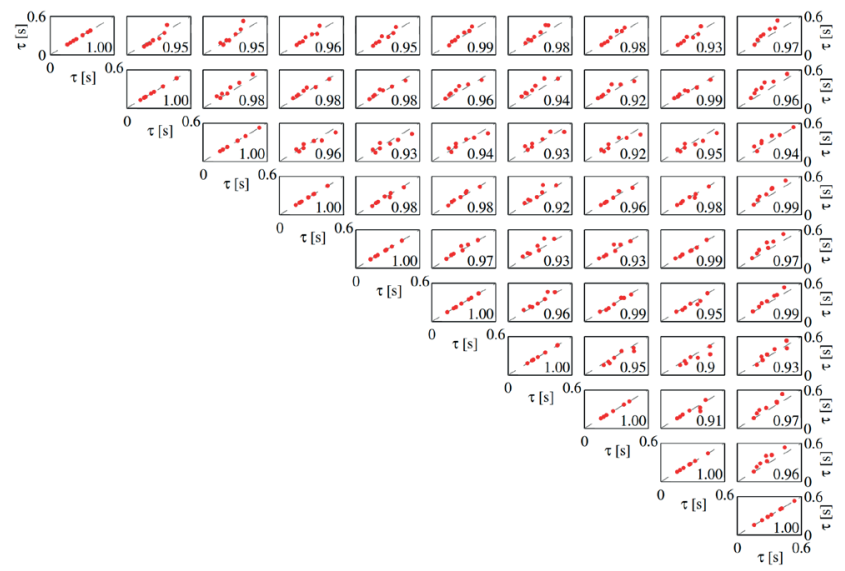

Fig. 15 Results and graphical demonstration of the linear correlation between the subjects

first and second test types are paired in the first row and the second column: Single LED - Button test versus Single LED - Pedal test. The horizontal coordinate of the 10 dots indicates the mean reaction time for each participant for the first test type in the subplot; while the meaning of the vertical coordinate is the same for the second test type. The linear autocorrelation is 1 in the diagonal; in that case the dots are located on the dashed line in the subplots.

The linear correlations of the pairs of the participants are shown in Table 4 and Fig. 15. Each row and column belongs to a participant. High correlation means that if a participant $i$ was fast in a certain test type, then participant $\mathrm{j}$ will be also fast in the same test, for any $\mathrm{i}-\mathrm{j}$ pairs. Each subplot in Fig. 15 corresponds to the correlation of two individuals. Since we accomplished 7 different types of tests, there are 7 points in each subplot. For instance, in the first row and second column, the first individual is compared to the second one. The horizontal coordinate of the 7 dots indicates the mean reaction time for every test

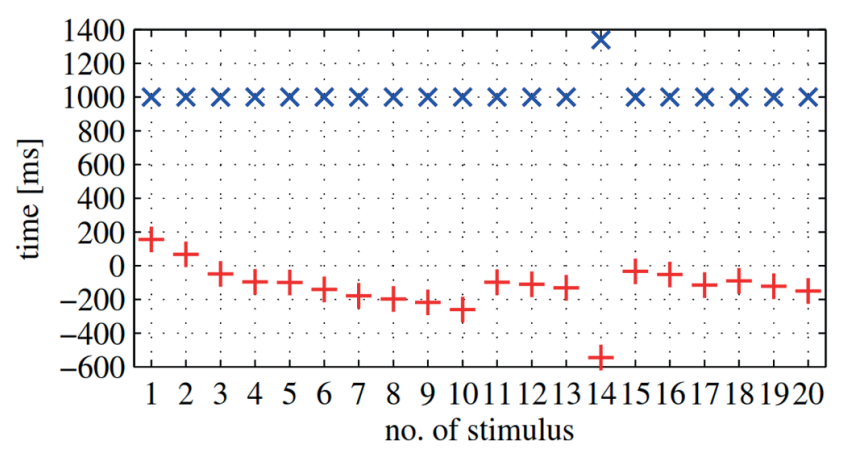

Fig. 16 Stimulus delay (blue $x)$, reaction time $($ red + ) for the series of 20 stimuli.

for the first participant in the subplot. The vertical coordinate of the 7 dots indicates the mean reaction time for every test for the second participant.

\subsection{Reaction time in case of rhythmic signals}

The result of one Periodic Beep - Button test session is presented in Fig. 16. The reaction time decreases quickly during first few reactions. Then the delay oscillates about negative $200 \mathrm{~ms}$ (advanced reaction). The $14^{\text {th }}$ stimulus was generated with a randomly set longer time period. The modified time period in this test was $1340 \mathrm{~ms}$. The reaction time after the $14^{\text {th }}$ reaction jumps down and then becomes a close-to-zero value again.

\section{Discussion}

The limitations of the present study were the low number of subjects and the low amount of measurement data. The standard deviation of the age of the subjects was relatively small; therefore it is not possible to derive conclusions regarding the population in general.

On the contrary, the capabilities of the CRTT were demonstrated and the reaction time was checked by 
oscilloscope. Thus the reaction time measured by the CRTT was verified by a certified instrument.

\subsection{Variety of reaction time tests}

The mean reaction time values in case of the different test types show significant difference. On the contrary, the reaction time for each test type was similar for different participants. The standard deviation was under the $20 \%$ of the mean value for all test types, except the $4^{\text {th }}$ and the $7^{\text {th }}$ test type (Single: Beep - Pedal and Pedals respectively), for which the standard deviation was still under the $30 \%$ of the mean value. If we consider all the 700 measurement data for all test types together, the standard deviation is much higher: $42 \%$ of the overall mean value. This supports that there is a significant difference between the mean reaction time measured in different test types.

The reaction time values are significantly larger (about 1.5 times) in the complex reaction time measurements comparing to the simple reaction test types. This phenomenon can be explained by the decision process required for the reaction $[8,20,21]$. One can also observe that in case of the single reaction time measurements, the single LED - Pedal test type results higher reaction time than LED - Button test type. The reason is that the length of the neural tissue is longer from the brain to the leg than from the brain to the hands. A further observation is that the reaction times are noticeably shorter in case of the beep stimulus compared to visual stimulus. The reason for this is the high amount of information required to process by the brain in case of visual sensing. The amount of information is much less in case of sound sensing. The above listed observations and results are in agreement with the literature data [8,20,21].

The correlation analysis of the tests shows that there is no remarkable correlation between the tests. It means that if someone performs well in test A than he/she is not necessarily fast in test B too. However, there is strong correlation between the subjects: if person $A$ is fast in a certain test and slow in another type of test, than the ratio of the reaction times for the same test types is similar to person B's ratio. The deduction is that there is no too much difference between the performances of people, but the difference is very high between the tests. This is promising when we think about further experimental and statistical study of the factors of reaction time.

\subsection{Reaction time in case of rhythmic signals}

The first 13 reactions in Fig. 16 show that the subject adapts to the periodically generated stimuli quite fast. The reaction became even negative, which means that the participant predicts the stimulus rather than reacting to it. It can be also observed in Fig. 16 that the participant could not adapt to the modified rhythm immediately (time period is changed from $1000 \mathrm{~ms}$ to $1340 \mathrm{~ms}$ ). The reaction was advanced with almost $600 \mathrm{~ms}$, which again shows that the subject predicted the next stimulus rather than reacting to it.

The index of the modified signal and the elongation of the time period were random parameters. Because of the random variables, large amount of experimental observations must be collected before deducing any general conclusion. The pattern can be varied too, for instance more than one modified time period could be included in a row or separately from each other; the overall rhythm could be changed after a random index without setting it back to the original rhythm. These different patterns will be studied in future work. The goal is the discovery of the adaptation and prediction capabilities of humans.

\section{Conclusion and outlook}

We demonstrated the capabilities of our newly developed Complex Reaction Time Tester instrument. 7 different test types were carried out with 10 participants and the measured data was analyzed statistically. Additionally, we demonstrated that the CRTT instrument is capable of testing human's reaction to rhythmic stimuli. However the effect of the pattern of the stimuli will be studied in future research by collecting a large amount of measurement data.

The two main novelties of the CRTT is that the reaction time can be measured in various combinations of the sensory organs and the reactions; and the CRTT is free of any uncertain time delay, in contrast to most of the available instruments. We showed that the accomplishment of the different test types requires the extension of the hardware with peripheral units and the extension of the control software in MatLab. However the hardware and the fundamental software components of the central unit do not have to be modified. We can conclude that the flexibility and versatility is the main strength of the CRTT, besides its high accuracy.

Additionally we mention that in some contexts, the nervous system does not seem to be interested in a short reaction time, but actually includes a long reaction time, referred to as the "central refractory time" [28]. When response times for a young healthy adult seem longer than expected for a simple reaction time test, then one might expect the possibility that a central refractory time might be involved. Note that the central refractory time hypothesis applies to 
complex tasks and requires that the brain must complete a motor plan before starting a new one. The CRTT would be ideally suited to explore these possibilities.

We believe that our Complex Reaction Time Tester instrument helps us to contribute to the systematic discovery of the factors that affects the reaction time. We expect that the effect of decision making and length of the involved neural tissues will be possible to clearly separate based on rigorous laboratory tests and statistical analysis.

\section{Acknowledgement}

This work was supported by The Higher Education Excellence Program of the Ministry of Human Capacities

\section{References}

[1] Stepan, G. "Delay effects in the human sensory system during balancing", Philosophical Transactions of the Royal Society A: Mathematical, Physical and Engineering Sciences, 367(1891), pp. 1195-1212, 2009.

https://doi.org/10.1098/rsta.2008.0278

[2] Milton, J. G., Insperger, T., Cook, W., Harris, D. M., Stepan, G. "Microchaos in human postural balance: Sensory deadzones and sampled time-delayed feedback", Physical Review E, 98(2), Article number: 022223, 2018.

https://doi.org/10.1103/PhysRevE.98.022223

[3] Matthews, P. B. C., Loewy, A. D., Haines, D. E., Lentz, T. L., Noback, C. R., Ratcliff, G., Rudge, P., Nathan, P. W. "Human nervous system", Encyclopædia Britannica, Encyclopædia Britannica, inc., 2019. Available at: https://www.britannica.com/science/ human-nervous-system [Accessed: 27 January 2019]

[4] Siciliano, B., Khatib, O. (eds.) "Springer Handbook of Robotics", Springer-Verlag, Berlin, Heidelberg, Germany, 2008. https://doi.org/10.1007/978-3-540-30301-5

[5] Galambos, P., Baranyi, P. "TP ${ }^{\mathrm{T}}$ Model Transformation: A Systematic Modelling Framework to Handle iInternal Time Delays in Control Systems", Asian Journal of Control, 17(2), pp. 486-496, 2015. https://doi.org/10.1002/asjc.1077

[6] Insperger, T., Milton, J., Stépán, G. "Acceleration feedback improves balancing against reflex delay", Journal of The Royal Society Interface, 10(79), Article number 20120763, 2013. https://doi.org/10.1098/rsif.2012.0763

[7] Pain, M. T. G., Hibbs, A. "Sprint starts and the minimum auditory reaction time", Journal of Sports Sciences, 25(1), pp. 79-86, 2007. https://doi.org/10.1080/02640410600718004

[8] Woods, D. L., Wyma, J. M., Yund, E. W., Herron, T. J., Reed, B. "Factors influencing the latency of simple reaction time", Frontiers in Human Neuroscience, 9, Article number 131, 2015. https://doi.org/10.3389/fnhum.2015.00131

[9] Zemkova, E., Vilman, T., Kováčiková, Z., Hamar, D. "Reaction Time in the Agility Test Under Simulated Competitive and Noncompetitive Conditions", Journal of Strength and Conditioning Research, 27(12), pp. 3445-3449, 2013. https://doi.org/10.1519/JSC.0b013e3182903222 in the frame of Biotechnology research area of Budapest University of Technology and Economics (BME FIKPBIO), by the Hungarian National Research, Development and Innovation Office (Project id.: NKFI-FK18 128636), by the National Research, Development and Innovation Fund (TUDFO/51757/2019-ITM, Thematic Excellence Program), by the MTA-BME Research Group on Dynamics of Machines and Vehicles and by the Hungarian-Chinese Bilateral Scientific and Technological cooperation Fund under Grant No. 2018-2.1.14-TET-CN-2018-00008.

[10] Mackenzie, I. S., Ware, C. "Lag as a determinant of human performance in interactive systems", iCHI '93 Proceedings of the INTERACT '93 and CHI '93 Conference on Human Factors in Computing Systems, Amsterdam, The Netherlands, 1993, pp. $488-493$. https://doi.org/10.1145/169059.169431

[11] Santos, V. G. F., Santos, V. R. F., Felippe, L. J. C., Almeida Jr., J. W., Bertuzzi, R., Kiss, M. A. P. D. M., Lima-Silva, A. E. "Caffeine Reduces Reaction Time and Improves Performance in SimulatedContest in Taekwondo", Nutrients, 6(2), pp. 637-649, 2014 https://doi.org/10.3390/nu6020637

[12] Firbank, M. J., O’Brien, J. T., Taylor, J. P. "Long reaction times are associated with delayed brain activity in lewy body dementia", Human Brain Mapping, 39(2), pp. 633-643, 2018. https://doi.org/10.1002/hbm.23866

[13] Quotronics Limited "Batak ${ }^{\mathrm{TM}}$ A Total Fitness Training Solution", 2017 [online] Available at: http://www.batak.com [Accessed: 24 January 2019]

[14] Fitlight Sports Corp. "Fitlight Trainer", [online] Available at: http:// www.fitlighttraining.com/ [Accessed: 24 January 2019]

[15] Topend Sports Network "SVT Reaction Time Test (AFL)", [online] Available at: https://www.topendsports.com/testing/tests/reaction-time-afl.htm [Accessed: 24 January 2019]

[16] Topend Sports Network "Reaction Time Gadgets", [online] Available at: https://www.topendsports.com/testing/products/ reaction-electronic.htm [Accessed: 24 January 2019]

[17] Goldberg, A., Schepens, S. L., Feely, S. M. E., Garbern, J. Y., Miller, L. J., Siskind, C.E., Conti, G. E. "Deficits in stepping response time are associated with impairments in balance and mobility in people with Huntington disease", Journal of the Neurological Sciences, 298(1-2), pp. 91-95, 2010. https://doi.org/10.1016/j.jns.2010.08.002

[18] Lafayette Instrument"MOART reaction and movement time panel with psymcon control", [online] Available at: http://lafayettelifesciences.com/products/moart-panel-with-control [Accessed: 24 January 2019] 
[19] Eckner, J. T., Kutcher, J.S., Richardson, J. K. "Effect of Concussion on Clinically Measured Reaction Time in 9 NCAA Division I Collegiate Athletes: A Preliminary Study", PM\&R Journal, 3(3), pp. 212-218, 2011. https://doi.org/10.1016/j.pmrj.2010.12.003

[20] Darbutas, T., Juodžbaliené, V., Skurvydas, A., Kriščiúnas, A. "Dependence of Reaction Time and Movement Speed on Task Complexity and Age", Medicina, 49(1), pp. 18-22, 2013. https://doi.org/10.3390/medicina49010004

[21] Arabaci, R., Çatikkaş, C., Çankaya, F., Şahin, S. Assessment body composition and leg reaction time of elite taekwondo athletes", Sport Sciences, 6(2), pp. 139-147, 2011. [online] Available at: http://dergipark.gov.tr/nwsaspor/issue/20137/213795 [Accessed: 24 January 2019]

[22] Spiteri, T., Cochrane, J. L., Nimphius S, "The Evaluation of a New Lower-Body Reaction Time Test", Journal of Strength and Conditioning Research, 27(1), pp. 174-180, 2013. https://doi.org/10.1519/JSC.0b013e318250381f

[23] Human Benchmark "Reaction Time Test", [online] Available at: https://www.humanbenchmark.com/tests/reactiontime [Accessed: 24 January 2019]
[24] Math is Fun "Reaction Time Test", [online] Available at: https:// www.mathsisfun.com/games/reaction-time.html [Accessed: 24 January 2019]

[25] Allen, J. "Red Light - Green Light Reaction Time Test", 2002, [online] Available at: https://faculty.washington.edu/chudler/java/ redgreen.html [Accessed: 24 January 2019]

[26] Kovács, B. A., Insperger, T. "Comparison of pixel-based position input and direct acceleration input for virtual stick balancing tests", Periodica Polytechnica Mechanical Engineering. (under review).

[27] multicomp "Z15G1307 Basic Z series micro switch - datasheet, [online] Available at: https://www.yumpu.com/en/document/ $\mathrm{read} / 17423637 / z 15 \mathrm{~g} 1307-z 15 \mathrm{~g} 1307-$ basic-z-series-micro-switchfarnell [Accessed: 24 January 2019]

[28] van de Kamp, C., Gawthrop, P. J., Gollee, H., Loram, I. D. "Refractoriness in Sustained Visuo-Manual Control: Is the Refractory Duration Intrinsic or Does It Depend on External System Properties?", PLoS Computational Biology, 9(1), Article number e1002843, 2013.

https://doi.org/10.1371/journal.pcbi.1002843 